\title{
Modeling the human knee joint using the Proper General- ized Decomposition
}

\author{
Chady Ghnatios ${ }^{1, *}$, Ilige Hage ${ }^{1, * *}$, and Najib Metni ${ }^{1, * * *}$ \\ ${ }^{1}$ Notre Dame Univresity-Louaize, department of mechanical engineering, Zouk Mosbeh, Lebanon
}

\begin{abstract}
Nowadays, human joints specifically movable are active research topics. The lack of effective replacements and the inefficient natural healing of these joints hinders any athlete from pursuing his career if injured in his joints. Therefore, researchers are testing innovative soft materials and biphasic materials as replacements of human joints. However, the lack of effective mechanical modeling is slowing the development of new replacements. In this work, we tackle the mechanical modeling of the synovial joint in a human knee. The tibiofemoral joint is modelled during impact. This joint is basically made of a cartilage, a meniscus (both a biphasic material) and the synovial fluid. The modeling is performed using Brinkman equation. However, the rich physics involved in the thickness direction requires a large number of degrees of freedom in the mesh to represent the physical phenomenon taking place in a knee joint. Thus, the use of model order reduction techniques appears to be an appealing approach in this situation. In fact, the proper generalized decomposition reduced the number of degrees of freedom by using domain decomposition. The result of this work shows the pressure and fluid flow in the synovial joint under impact. A post treatment of the solution estimates the force held by each of the fluid and solid components of the cartilage joint. This model could be used to the human knee to estimate its components' velocities and pressure fields while performing an activity.
\end{abstract}

\section{Introduction}

The knee is a type of synovial joint or a modified hinge joint. Its three functional compartments permit flexion and extension as well as slight internal and external rotation. These compartments make the knee a complex joint, which is vulnerable to a variety of injuries such as fractures, dislocations, sprains, and ligament tears that could be healed through rehabilitation exercises or may lead to surgery for correction.

This study focuses on modeling the knee joint under vertical impact and the derived model can be used for any activity. The tiobiofemoral joint is modeled in order to analyze the distribution of forces inside the joints and ligaments and thus determine the risk level for injury. This study models the tibia and the femur and their corresponding articular cartilage as well as the meniscus as two biphasic cylinders. In between those two lies a synovial fluid that dampens the contact between them.

Biphasic materials are made of a fluid component in a solid mesh with low permeability, pressurizing the fluid and holding the fluid flow out of the joint [1]. Therefore the fluid pressurization is believed to be one of the most important load carrying mechanisms of the joint [2,3]. Moreover, modeling of biphasic material is not mastered yet. Recent works have focused on modeling biphasic materials, human cartilage and their mimics, using advanced numerical or analytical techniques $[4,5]$. However, the rich physics in the thickness direction (pressure discontinuity, presence of solid calcium brushes...) requires a tremendous number of degrees of freedom to reproduce with high fidelity the involved physics. Therefore, using the Proper Generalized Decomposition (PGD) model order reduction technique appears as an appealing approach. Many model order reduction techniques exist, all based on domain

\footnotetext{
*e-mail: cghnatios@ndu.edu.lb

**e-mail: ilige.hage@ndu.edu.lb

***e-mail: nmetni@ndu.edu.lb
} 
decomposition, however the PGD is the only one qualified as an "a priori" model order reduction technique $[6,7]$.

The use of the PGD in this work will allow the simulation of the full $3 D$ domain as a sequence of $2 D \times 1 D$ problems $[8,9]$. We model the knee joint using the Brinkman equation as detailed in section 2, taking into consideration the cartilage brush permeability, later on we show the simulation results clearly illustrating the pressure discontinuity on the domain interface.

\section{Modeling the knee joint}

Biphasic materials can be modeled using the Brinkman equations [10]. Neglecting the porosity changes, the Brinkman equations can be written as:

$$
\left\{\begin{array}{l}
\nabla(\eta \nabla \mathbf{v})-\frac{\mu}{\mathbf{K}} \mathbf{v}-\nabla P=0 \\
\nabla \cdot \mathbf{v}=0
\end{array}\right.
$$

Where $\mathbf{v}$ is the velocity, $P$ the fluid pressure, $\mu$ the fluid viscosity, $\eta$ the dynamic effective viscosity and $\mathbf{K}$ the effective permeability. For lack of exact definition of the LadyzenskajaBabuska-Brezzi conditions, also knwon as the $L B B$ conditions, for a mixed formulation in a separated representation framework [11], we use the penalty formulation to solve the problem using the PGD, thus we write:

$$
\nabla \cdot \mathbf{v}=\lambda P
$$

Where $\lambda$ is a small enough penalty term. Combining equations (1) and (2) leads to the following final strong form of the problem:

$$
\nabla(\nabla . \mathbf{v})+\lambda \nabla(\mu \nabla \mathbf{v})-\frac{\lambda \cdot \mu^{\prime}}{K} \mathbf{v}=0
$$

Equation (3) combines the Stokes equation at high permeability in the fluid region, and Darcy's equation for the low permeability cartilage region as illustrated in figure 1 .

The PGD already made its proof in non prismatic domains [12, 13], however for sake of simplicity we are considering the knee synovial joint as a cylindrical domain with horizontal plane of symmetry. The final simulation domain is illustrated in figure 1, where the calcium brush represents the carticlage and the meniscus, and $u$ is half the imposed velocity, found using the conservation of momentum during the impact. The solution is seeked in a separated representation such as:

$$
\mathbf{v}=\left(\begin{array}{rl}
u & =\sum_{i=1}^{i=N} X_{u i}(x, y) \cdot Z_{u i}(z) \\
v & =\sum_{i=1}^{i=N} X_{v i}(x, y) \cdot Z_{v i}(z) \\
w & =\sum_{i=1}^{i=N} X_{w i}(x, y) \cdot Z_{w i}(z)
\end{array}\right)
$$

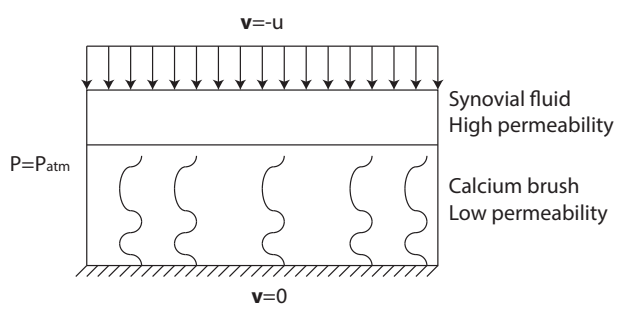

Figure 1. The simulated domain after considering the middle plane as a plane of symmetry

$u, v$ and $w$ being the $x, y$ and $z$ components of the velocity vector $\mathbf{v}$. Once the velocity $\mathbf{v}$ is known, we can find the pressure using equation (2) [14, 15]. Figure 2 illustrates the magnitude of the velocity field in a cross section where the upper cylinder represents the fluid ans the lower represents the biphasic material, while figure 3 illustrates the pressure fields in a vertical section in the knee joint, for $u=1 \mathrm{~mm} / \mathrm{s}$. The mesh consists of 2269 nodes in the in - plane dimension and 1000 nodes out-of-plane, equivalent to 9,076,000 $3 \mathrm{D}$ degrees of freedom. The problem is solved within 6.45 seconds on a normal portable PC using Matlab®. Figures 2 and 3 clearly illustrate the discontinuity in the velocity and pressure fields between the synovial fluid and the cartilage. 

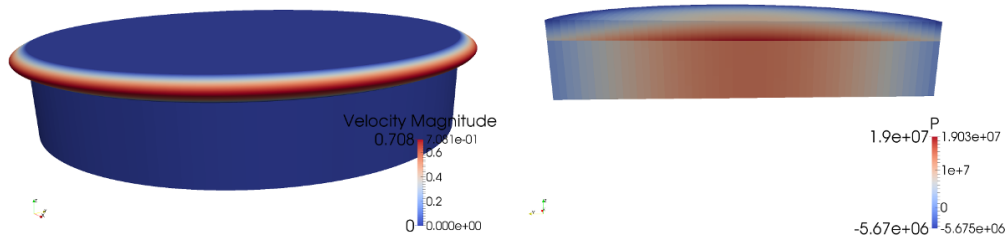

Figure 2. The magnitude of the velocity $\mathbf{v}(\mathrm{mm} / \mathrm{s})$ Figure 3. The pressure field $P(\mathrm{~Pa})$ in a vertical in the deformed shape just after the impact section in the knee joint

\section{Conclusion}

The illustrated model shows the velocity fields, deformation and pressure inside the tibiofemoral joint. The result can be post treated to estimate potential injury levels due to applied forces. Moreover, the magnitude of the pressure can be found using the imposed force as an extra coordinate of the problem to estimate a maximum possible applied load to avoid injury. The work consists an innovation in modeling biphasic soft materials such as the human joints or their mimcs. The use of the PGD allows high fidelity representation of the results, while dramatically reducing the calculation time as shown in the illustrated example.

\section{Acknoledgement}

This work is part of the CNRS-L and NDU grant for the project entitled: Human joints impact measurement and optimization.

\section{References}

[1] R.M. Espinosa-Marzal, R.M. Bieleckia, N.D. Spencer, Soft matter 9, 10572 (2013)

[2] C. Ghnatios, C.H. Mathis, R. Simic, N.D. Spencer, F. Chinesta, Soft Matter 13, 4482 (2017)

[3] C. Ghnatios, C. Mathis, F. Chinesta, Poroelastic properties identification through mico indentation modeled by using the proper generalized decomposition, in 3rd International Conference on Advances in Computational Tools for Engineering Applications (ACTEA) (IEEE, 2016), pp. 141-145

[4] T. Sherstova, B.T. Stokke, B. Skallerud, G.M. abd Victorien Emile Port, Soft matter 12, 7338 (2016)

[5] Y. Shi, D. Xiong, Wear 305, 280 (2013)

[6] C. Ghnatios, Ph.D. thesis, Ecole Centrale Nantes (2012)

[7] J.V. Aguado, D. Borzacchiello, C. Ghnatios, F. Lebel, R. Upadhyay, C. Binetruy, F. Chinesta, Advanced Modeling and Simulation in Engineering Sciences 4, 1 (2017)

[8] E. Cueto, C. Ghnatios, F. Chinesta, N. Monte, F. Sanchez, A. Falco, Key Engineering Materials 611, 339 (2014)

[9] N. Bur, P. Joyot, C. Ghnatios, P. Villon, E.C. Cueto, F. Chinesta, Advanced Modeling and Simulation in Engineering Sciences 3, 1 (2016)

[10] C. Ghnatios, F. Chinesta, C. Binetruy, International Journal of material forming 8, 73 (2015)

[11] C. Ghnatios, R. Himo, E. Hachem, A stabilized PGD mixed formulation of the NavierStokes equation, application to a lid-driven cavity, in ECCM-ECFD (Glasgow, 2018), pp. 1-11

[12] C. Ghnatios, G. Xu, A. Leygue, M. Visionneau, F. Chinesta, A. Cimetiere, Discrete \& Continuous Dynamical Systems-Series S 9, 475 (2016)

[13] C. Ghnatios, A. Ammar, A. Cimetiere, A. Hamdouni, A. Leygue, F. Chinesta, First Steps in the space separated representation of models defined in complex domains, in ASME ESDA 2012, 11th Biennial Conference on Engineering Systems Design and Analysis (2012), pp. 37-42

[14] T.J. Hughes, W.K. Liu, A. Brooks, Journal of computational Physics 30, 1 (1979)

[15] T. Hughes, The finite element method: linear static and dynamic finite element analysis (Dover Publications, NY, Englewood cliffs, NJ, 2000) 